\title{
Angelika Linke \& Juliane Schröter (Hg.). 2017. Sprache und Beziehung (Linguistik - Impulse \& Tendenzen). Berlin, Boston: De Gruyter Mouton. vii, 448 S.
}

Besprochen von Konstanze Marx: Institut für Deutsche Sprache Mannheim, R5, 6-13, D-68161 Mannheim, E-Mail: Konstanze.Marx@ids-mannheim.de

https://doi.org/10.1515/zrs-2018-0002

Das hier $\mathrm{zu}$ besprechende Buch, das Ergebnisse einer gleichnamigen Tagung zusammenfasst, die im Juni 2013 in Zürich stattfand, macht eines offenkundig: Wer in jenem Sommer nicht dabei war, hat etwas verpasst. Umso glücklicher darf man sein, dass Angelika Linke und Juliane Schröter die Arbeit, die mit der Herausgabe eines Sammelbandes verbunden ist, auf sich genommen haben. Mehr noch: In einem programmatischen ersten Kapitel geben sie einen systematischen Einblick in das tragfähige Forschungsfeld „Sprachliche Relationalität“ (vgl. S. 1-6), das ganz im Sinne der emotiven Wende in der Sprachwissenschaft konkrete theoretische Anschlussfähigkeit signalisiert, wo bislang eine „fast unübersehbare Menge an Veröffentlichungen“ (Schwarz-Friesel 2013: 16) zwar zeigte, wie attraktiv die Thematik ist, aber auch wie unstrukturiert sich die Zuwendung dazu gestaltet. Dass der Band nun weitere „exemplarische Besetzungen“ (S. 21) des Forschungsfeldes zur Diskussion stellt, wird hier keinesfalls als Nachteil angesehen, sondern als methodisch folgerichtiger empirischer Zugang zur Erschließung eines Forschungsfeldes unter den zielsetzenden Leitfragen „Wie werden im Medium von Sprachgebrauch und Sprache Konzeptualisierungen, Kategorisierungen und Differenzierungen menschlicher Beziehungen ausgebildet, verfestigt und auch wieder verändert?“ und „Welche sprachgeformten Beziehungskonzepte, -kategorien und -unterschiede sind typisch für bestimmte historische Epochen bzw. für bestimmte soziale Gruppierungen?“ (S. 21)

Die Herausgeberinnen zeigen auf, dass sowohl sprachtheoretische Überlegungen, Modelle sprachlicher Grundfunktionen, interaktionale, pragmatische sowie text- und gesprächslinguistische Zugänge, Höflichkeitsforschung und Nähe- und Distanz-Ansätze, lexikalische wie auch diskursorientierte Zugriffe die Relationalität von Sprache berühren. Beziehung wird hier als „konkrete Relation zwischen (mindestens zwei) Menschen“ (S. 15) aufgefasst, die ein performatives (manifeste Interaktionspraxis) und ein reflexives (Deutung der gemeinsamen Interaktion) Element aufweist. Die skizzierten drei Untersuchungsperspektiven (Wie prägt sich Sprache in Beziehungen aus? Wie werden Beziehungen in Sprache [aus-]gebildet? Welche kulturellen Muster sind erkennbar?) werden denn auch im vorliegenden Band sichtbar und an facettenreichen, einschlägigen Unter- 
suchungsgegenständen expliziert. Das liest sich wie ein umfangreiches Werbeprogramm für die Sprachwissenschaft und die folgende Zusammenfassung der Beiträge darf denn auch als Werbung für das Buch verstanden werden.

Horst J. Simon stellt in seinem struktur-onomasiologisch ausgerichteten Beitrag menschliche Beziehungen vor, die sprachstrukturell markiert werden. Dazu gehört die Beteiligtenrelation, was u.a. die Frage involviert, ob ein Adressat der Referenzmenge angehört, die den Sprecher inkludiert, und sich in sogenannten ,Schwiegermuttersprachen' (in Australien) so äußert, dass die Belegung einer Rolle eines in Tabu-Relation stehenden Mithörers systematisch differierend kodiert wird. Dazu gehören Generationendistanzen, wenn das relative Alter von Sprecher und Adressat in Anredeformen Eingang findet und den unterschiedlichen Zugriff auf grammatische Mittel motiviert. In diesem Zusammenhang sind Verwandtschaftsverhältnisse (z.B. auch das Angeheiratetsein) generell zu erwähnen, die grammatisch und systematisch-lexikalisch wirksam werden. Im Text von Christa Dürscheid geht es um Online-Strategien der Anbahnung menschlicher Beziehungen. Der Fokus liegt dabei auf der sogenannten Courtship-Kommunikation (Murstein 1972), die hier am Beispiel von Parship betrachtet wird. Als wichtigen Aspekt hebt die Autorin die formal-sprachliche Gestalt der Texte hervor und schreibt insbesondere der Orthographie eine sogenannte ,Gatekeeping'-Funktion zu. Sie regt damit zur empirischen Untersuchung dieser interessanten Hypothese an. Nicole Müller \& Joachim Scharloth stellen rekurrente Muster und ihre typischen Verkettungen in Erzählungen vom ,Ersten Mal' vor. Anders als die Analysen der anderen Beiträge basiert diese Untersuchung auf einem umfangreichen Datenkorpus. Auf diese Weise ließ sich ein narratives Script extrahieren, das sich aus einer Exposition (mit Nennung des Alters, der Beziehungsdauer und Partner), der Komplikation (Verabredung und Herstellung von Einverständnis), Lösung (Vorspiel und Akt), Evaluation (Bewertung des subjektiven Empfindens) und Coda (Grüße, Liebeserklärung, Ratschläge) zusammensetzt und damit Elemente alltagssprachlicher Erzählungen bedient (vgl. Labov \& Waletzky 1967). Interessant dabei ist, dass die Darstellung des Sexualakts sprachlich auffallend stereotyp realisiert wird und in die Darstellung einer Verbundenheit durch romantische Liebe eingebettet wird, wodurch die Erzählungen eine „sexualmoralische Veredelung“ (S. 95) erfahren. Damaris NüBling nähert sich „intrinsisch forschungsaversiven“ (Hirschauer 2012, zitiert im Beitrag) intimen Beziehungen über Spitznamen, die der „onymischen Fellpflege“ (S. 101) dienen. Dabei stellt sie heraus, dass Spitznamen primär dazu dienen, eine Nähebeziehung zu etablieren, die mit dem/der NamensträgerIn nicht verhandelt wird und dadurch Macht und Kontrolle markiert. Geschlechtsspezifische Unterschiede bei Kosenamen schlagen sich z.B. in der Form nieder, wenn männliche Kosenamen aus Nachnamen, weibliche Kosenamen hingegen aus Vornamen abgeleitet werden; im Alter (so 
scheinen jüngere Kinder häufiger Kosenamen zu verwenden) und in der Häufigkeit (Mädchen scheinen häufiger Kosenamen zu bekommen). Innerhalb von Paarbeziehungen scheint die reziproke Verwendung von Kosenamen eine Gleichheit der PartnerInnen zu indizieren. Koseformen spielen auch im anschließenden Beitrag von Susanne Günthner \& Qiang Zhu eine Rolle, der sich mit kulturspezifischen Adressierungen in SMS-Interaktionen als „kommunikative[n] Praktiken zur Konstruktion, Verfestigung und Modifikation sozialer Beziehungen“ (S. 119) auseinandersetzt. Während die Verwendung von Verwandtschaftstermini in deutschen SMS-Interaktionen eher scherzhaft oder ironisch ist, scheint sie in chinesischen SMS einer Norm zu folgen, weil es als Tabu gilt, höherstehende oder ältere Verwandte mit dem Vornamen anzureden. Anders als im Deutschen stellt auch die Anrede der Kinder als Sohn oder Tochter den unmarkierten Fall dar.

Um Anrede geht es auch im Beitrag von Anja Stukenbrock \& Cornelia Bahr, hier allerdings um den Gebrauch des generischen $d u$ in Abgrenzung zum deiktisch-symbolischen und deiktisch-gestischen Gebrauch des Pronomens. Die Autorinnen zeigen typische grammatische Kontexte, systematische Restriktionen und interaktionale Aspekte auf, die die Referenzialisierung erleichtern. Den Übergang zum diachron ausgerichteten Teil des Bandes markiert Hans-Peter Schifferles lexikographisch-lexikologische Studie $\mathrm{zu}$ den schweizerdeutschen relationalen Personenbezeichnungen des mundartlichen Gschpäändli, des Helvetismus Koleege und des geschlechtsspezifisch restriktiv gebrauchten Fründ. Dessislava Stoeva-Holm diskutiert Freundschaft mit deskriptivem Blick auf die kinderkulturelle Austauschpraxis von Poesiealben. Eintragungen setzen sich zusammen aus Anreden, Widmungen und Reproduziertexten, die insbesondere bei Kindern gereimte Formelhaftigkeit kennzeichnet. Die interessante Beobachtung, dass Eintragungen häufig in Abschiedssituationen erbeten werden, hätte auch zu einer kritischen Betrachtung der Qualität der jeweiligen zwischenmenschlichen Beziehung führen können, die sich in einer Art „Buchführung“ (S. 228) niederschlagen.

Eine noch deutlich reduziertere Form des sprachlichen Ausdrucks menschlicher Beziehungen zeigt sich in Traueranzeigen im Nationalsozialismus. JöRg RIECKE macht anhand von Kommentaren aus den Tagebüchern Viktor Klemperers und Friedrich Kellners nachvollziehbar, dass Todesanzeigen trotz ihrer ausgeprägten Formalisierung das Potenzial aufweisen, die Positionierung der Trauernden zum Staat zu vermitteln. Umgesetzt wird dies etwa durch die Abschwächung oder sogar Vermeidung von typischen Formeln, wie z.B. für Führer und Vaterland. Einen Schritt weiter zurück in der Geschichte geht BRITT-MARIE Schuster, die Fahrtenberichte der Bündnischen Jugend der 1920er Jahre betrachtet und damit quasi nebenbei ein bislang ungeschriebenes Kapitel in der Jugendsprachforschung erschließt. Als Merkmale einer Stände- und Klassen-, aber auch 
Geschlechterunterschiede nivellierenden jugendkulturellen Schriftpraxis arbeitet sie an einem Fallbeispiel auktorialen Erzählstil, fingierte innere Monologe, die Technik des Verrätselns, Referenzen auf die Gruppe oder rituelle Sequenzen, wie z.B. Begrüßungen und Verabschiedungen, heraus. Grüße nun werden von Juliane SCHRÖTER in den Mittelpunkt gerückt, wenn sie diese Korrespondenzschlüsse als besondere Form der sprachlichen Beziehungsgestaltung ausdeutet. So signalisiert der sprachliche Akt des Grußübermittelns eine Zugehörigkeit zu einem sozialen Netz und kann die Beziehung von einer dritten Person zum Briefempfänger fördern, aber gleichzeitig auch die Beziehung zwischen dem Briefverfasser und dem Briefempfänger stärken.

Der folgende Beitrag von KlaAs-Hinrich Ehlers greift die Textsorte Brief des 19. und 20. Jahrhunderts erneut auf und problematisiert die Beziehungsrelevanz der Selbstbezeichnung. Zu diesem Zweck werden semiprofessionelle Stilempfehlungen von Sprachratgebern zu privater und geschäftlicher Korrespondenz ausgewertet. Ehlers beschreibt, wie sich Anforderungen an Titulatur, Unterschrift und Verwendung des Pronomens ich im Verlauf der Zeit (bis ins 21. Jahrhundert) wandelten und Statusmarkierungen durch ästhetische respektive praktische Kriterien abgelöst wurden. Als methodisches Problem stellt Matrhias Schulz die Untersuchung von Kosenamen des 19. und 20. Jahrhunderts heraus. Er wertet einerseits ein 126 Lexeme und entsprechende lexikographische Angaben umfassendes Korpus aus, das Ergebnis einer Wörterbuchrecherche zu schmeichel und kose ist, und sucht andererseits nach heute belegten Kosenamen im deutschen Textarchiv. Dabei stößt er auf Bezeichnungen wie Schatz oder Schnuffel, die schon lange gebräuchlich sind. Susanne Tienken beschreibt in ihrem Beitrag Stammbücher als beziehungskonstitutive Gattungen und knüpft mit ihrer Konzeption des Genres als soziales Handeln an einen Textsortenbegriff an, der „,von einer Kommunikationsgemeinschaft diskursiv hervorgebracht“ (Luginbühl \& Perrin 2011: 577) wird. Im 17. und 18. Jahrhundert wurden kleinformatige Alben von Gelehrten, Studenten und Adeligen auf Reisen mitgeführt, bildeten jedoch nur den Kreis gesellschaftlich gleichrangiger oder höherstehender Personen ab. Dabei wird die soziale Ordnung in der Organisation des Albums gespiegelt. An Studentenstammbüchern werden misogyne Motive als Mittel zur Konstruktion der chronotopischen Beziehung von Studenten (2. Hälfte 18. Jhdt.) herausgearbeitet. Anja Lobenstein-Reichmann wählt einen lexikographischen Zugang, um Beziehungswirklichkeiten des Frühneuhochdeutschen zu rekonstruieren. Sie zeigt an Ausdrücken wie band, heim, geselschaft, kunde oder gunst, dass sich die jeweiligen zeitgenössischen Macht-, Hierarchie-, Besitz- und Zugehörigkeitsverhältnisse sowie die besondere Bedeutung räumlicher Nähe und zeitlicher Kontinuität im Wortschatz abbilden. Den Abschluss des Bandes bildet eine Interpretation frühneuzeitlicher Ehegerichtsakten der Historikerin Francisca LoETz, die eine Reflexion methodologischer Fra- 
gen an der Schnittstelle zwischen Linguistik und Geschichtsschreibung integriert. An Fällen wie der Magd Elßa Gesslerin, der es gelingt, die Heirat mit dem Bruder ihres Dienstherrn einzuklagen, nachdem er um sie gebuhlt hatte, oder der Eheleute Trindler, deren Ehekonflikt auf eine neun Jahre andauernde Affäre Felix Trindlers mit einer Witwe zurückzuführen ist, wird gezeigt, wie ein historischer Zugang die sprachliche Analyse gewinnbringend erweitern und zugleich auch von ihr profitieren kann.

An dieser Inhaltswiedergabe werden zwei Dinge deutlich: Die meisten Beiträge stützen sich (1) auf eine nur schmale Datenbasis, was von den AutorInnen selbst kritisch reflektiert und problematisiert wird, vgl. etwa die Kategorisierung als „Jäger- und Sammlerforschung“ bei Horst J. Simon (S. 45). Somit lese ich die Texte als beobachtende Teilnehmerin einer inspirierenden Exploration des Forschungsfeldes. Die gegenwartsbezogenen Beiträge sind (2) unterrepräsentiert und damit kommt aus meiner Sicht auch die medial vermittelte Beziehungskonstruktion und -reflexion zu kurz, die zu Beginn des Buches ausdrücklich als Untersuchungsgegenstand definiert wird. Wichtig ist mir hier nicht der Blick auf neue Medien als beziehungszersetzende und beziehungskonstitutive Ressource. Der letzte Aspekt wird nur in zwei Beiträgen skizziert. Wenn das Thema in anderen Kapiteln gestreift wird, geschieht es oberflächlich und klingt gezwungen, was jedoch zeigt, wie viel Potential dieses neue Feld für die künftige Forschung bereithält. An einigen Stellen hätte ich mir Querverweise auf Beiträge im Buch gewünscht, auch weil sich die Herausgeberinnen für eine chronologische Ordnung der diachron ausgerichteten Beiträge entschieden haben. Abschließend darf ich bemerken, dass sich beim Lesen eine Erkenntnis geradezu aufdrängt, die im Kontext des neuen Forschungsfeldes ebenfalls eine genauere Untersuchung wert wäre: die Relationalität der Textsorte Rezension, eines offenen Review-Verfahrens also, das den/die RezensentIn in einen Konflikt zwischen notwendiger sachlicher Kritik (vgl. Stegert 1997) und wohlgesinnter Verbundenheit mit den KollegInnen stürzen kann. Die ausgesprochen hohe Qualität des vorliegenden Buches hat mich vor diesem Dilemma bewahrt.

\section{Literatur}

Labov, William \& Joshua Waletzky. 1967. Narrative analysis. In: June Helm (Hg.): Essays on the Verbal and Visual Arts. Seattle: University of Washington Press, 12-44. Wiederabdruck in: Journal of narrative and life history 7/1997, 3-38.

Luginbühl, Martin \& Daniel Perrin. 2011. „,das, was wir in der Tagesschau den Rausschmeißer nennen': Altro- und Ethnokategorisierung von Textsorten im Handlungsfeld journalistischer Fernsehnachrichten“. In: Stephan Habscheid (Hg.): Textsorten, Handlungsmuster, 
Oberflächen. Linguistische Typologien der Kommunikation. Berlin, New York: De Gruyter, 577-596.

Murstein, Bernard. 1972. Person Perception and Courtship Progress among Premarital Couples. In: Journal of Marriage and the Family 34, 621-626.

Schwarz-Friesel, Monika. 2013. Sprache und Emotion. 2. Aufl. Tübingen: Narr/Francke/Attempto. Stegert, Gernot. 1997. Die Rezension: Zur Beschreibung einer komplexen Textsorte. In: Beiträge zur Fremdsprachenvermittlung 31, 89-110. 